\begin{tabular}{|c|c|}
\hline Title: & $\begin{array}{l}\text { Comparison of Synchronous Machine Designs with Displaced Reluctance Axis } \\
\text { Considering Losses and Iron Saturation }\end{array}$ \\
\hline Authors: & Patrick Winzer, Martin Doppelbauer \\
\hline Institute: & $\begin{array}{l}\text { Karlsruhe Institute of Technology (KIT) } \\
\text { Elektrotechnisches Institut (ETI) } \\
\text { Hybrid Electric Vehicles (HEV) }\end{array}$ \\
\hline Type: & Conference Proceedings \\
\hline Published at: & $\begin{array}{l}\text { Proceedings } 2015 \text { International Electric Machines and Drives Conference (IEMDC), } \\
\text { Coeur D'Alene, Idaho, USA, May 10-13, } 2015 \\
\text { Publisher: IEEE, Piscataway (NJ) } \\
\text { Year: } 2015 \\
\text { ISBN: } 978-1-4799-7941-7 \\
\text { Pages: } 1801-1807\end{array}$ \\
\hline Hyperlinks: & DOI: https://doi.org/10.1109/IEMDC.2015.7409308 \\
\hline
\end{tabular}

(C) 2015 IEEE. Personal use of this material is permitted. Permission from IEEE must be obtained for all other uses, in any current or future media, including reprinting/republishing this material for advertising or promotional purposes, creating new collective works, for resale or redistribution to servers or lists, or reuse of any copyrighted component of this work in other works. 


\title{
Comparison of Synchronous Machine Designs with Displaced Reluctance Axis Considering Losses and Iron Saturation
}

\author{
Patrick Winzer and Martin Doppelbauer
}

\begin{abstract}
Synchronous machines with displaced reluctance axis promise improved performance due to the optimized utilization of reluctance and synchronous torque. Their torque characteristics make these machines interesting for applications which mostly require just one operation mode, i.e. motoring or generating, such as traction drives for electric vehicles. First of all, this paper shows that arbitrarily displaced reluctance axes are difficult to realize with pure permanent magnet and pure electrically excited synchronous machines and yield disadvantageous machine properties. In the second part, a hybrid synchronous machine topology which incorporates a displaced reluctance axis is introduced and its conceptual advantages compared to conventional machine designs are shown.
\end{abstract}

Index Terms-Adjustable speed drive, Efficiency, Electric vehicles, Electrical drive, Hybrid excitation machine, Optimization, Permanent magnet machine, Synchronous machine

\section{INTRODUCTION AND THEORETICAL BACKGROUND}

Modern control schemes for synchronous machines, such as the maximum torque per ampere (MTPA) strategy [1], were discovered almost 30 years ago. They use the saliency of the rotor geometry to generate the greatest possible torque as a combination of the reluctance torque and the synchronous torque. It was found that the actual design of the machine, i.e. its distinct reluctance and permanent magnet nature, determines the specific torque versus speed characteristic.

Consequently, normalized parameter planes for machine design have been developed for both permanent magnet synchronous machines (PMSM) [2], which exhibit $L_{\mathrm{d}}<L_{\mathrm{q}}$ and salient pole synchronous machines (SPSM) [3], which have $L_{\mathrm{d}}>L_{\mathrm{q}}$. These planes are depending on the saliency $\zeta=\frac{L_{\mathrm{q}}}{L_{\mathrm{d}}}$ and the normalized permanent magnet (PM) flux linkage $\psi_{\mathrm{PM}}$ and are useful for determining optimum machine parameters for a desired operating performance.

If the current angle $\gamma$ is varied, which is defined as the angle between the $\mathrm{q}$ axis and the stator current space vector, the amounts of synchronous torque and reluctance torque change as well. However, the current angle $\gamma$ which yields the greatest synchronous torque is not identical to the current angle $\gamma$ which yields the greatest reluctance torque for these machines

P. Winzer is with the Institute of Electrical Engineering (ETI) - Hybrid Electric Vehicles (HEV), Karlsruhe Institute of Technology (KIT), 76128 Karlsruhe, Germany (e-mail: patrick.winzer@kit.edu)

M. Doppelbauer is head of the department Hybrid Electric Vehicles (HEV) at the Institute of Electrical Engineering (ETI), Karlsruhe Institute of Technology (KIT)

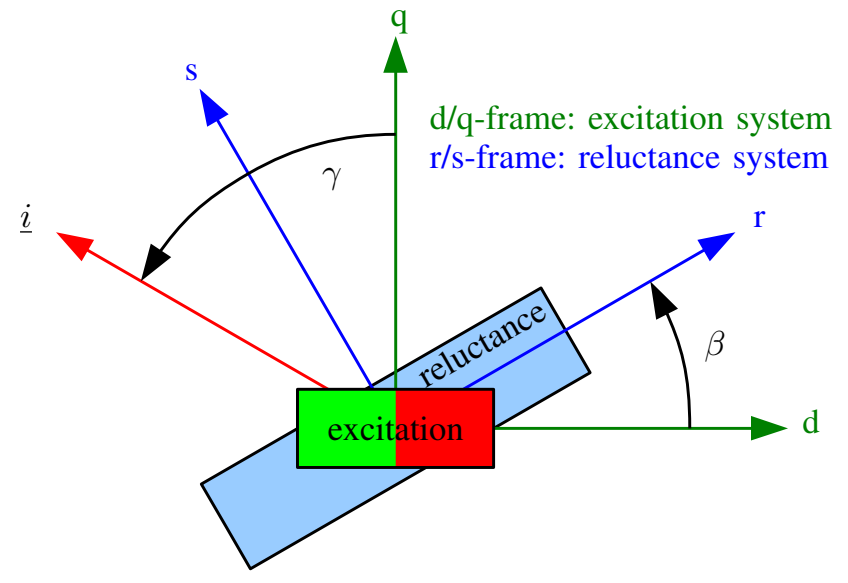

Fig. 1. Basic reference frames of machines with displaced reluctance axis and the definition of the displacement angle $\beta$ and the current angle $\gamma$ [4].

[5]. To overcome this disadvantageous characteristic, a theoretical analysis has been carried out in [4] to investigate machines with displaced reluctance axis, see Fig. 1. These machines can have any arbitrary angle $\beta$ between the excitation axis $\mathrm{d}$ and the reluctance axis $r$. The reluctance axis $r$ is the axis where the greatest inductance $L_{\mathrm{r}}$ appears, whereas the excitation axis is the one where the spatial maximum of the rotor flux is located.

Using this representation, conventional PMSM with $L_{\mathrm{d}}<$ $L_{\mathrm{q}}$ have $\beta=90^{\circ}$ (i.e. $L_{\mathrm{r}}=L_{\mathrm{q}}$ ), SPSM machines with $L_{\mathrm{d}}>L_{\mathrm{q}}$ have $\beta=0^{\circ}$ (i.e. $L_{\mathrm{r}}=L_{\mathrm{d}}$ ). It was shown that, depending on the saliency $\zeta=\frac{L_{\mathrm{r}}}{L_{\mathrm{s}}}$ of the machine and the normalized permanent magnet (PM) flux linkage $\psi_{\mathrm{PM}}$, there is a displacement angle $\beta$, which leads to optimum motor performance. Fig. 2 shows an exemplary plot of the normalized $\psi_{\mathrm{PM}}-\beta$-parameter plane with a constant saliency of $\zeta=4$ for the normalized maximum motoring torque $t$ as a function of the normalized PM flux linkage $\psi_{\mathrm{PM}}$ and the displacement angle $\beta$. An initial conventional design is marked by the blue cross at position $\mathrm{A}$. An angle of $\beta \approx 60^{\circ}$ leads to the greatest torque for a fixed value of $\psi_{\mathrm{PM}}$ (red cross at position B), or to the lowest required value of $\psi_{\mathrm{PM}}$ for the same torque respectively (gray cross at position C). Due to the magnetic asymmetry, performance in generating mode deteriorates noticeably, however, this can be accepted for a lot of applications such as electric traction, as the generating 


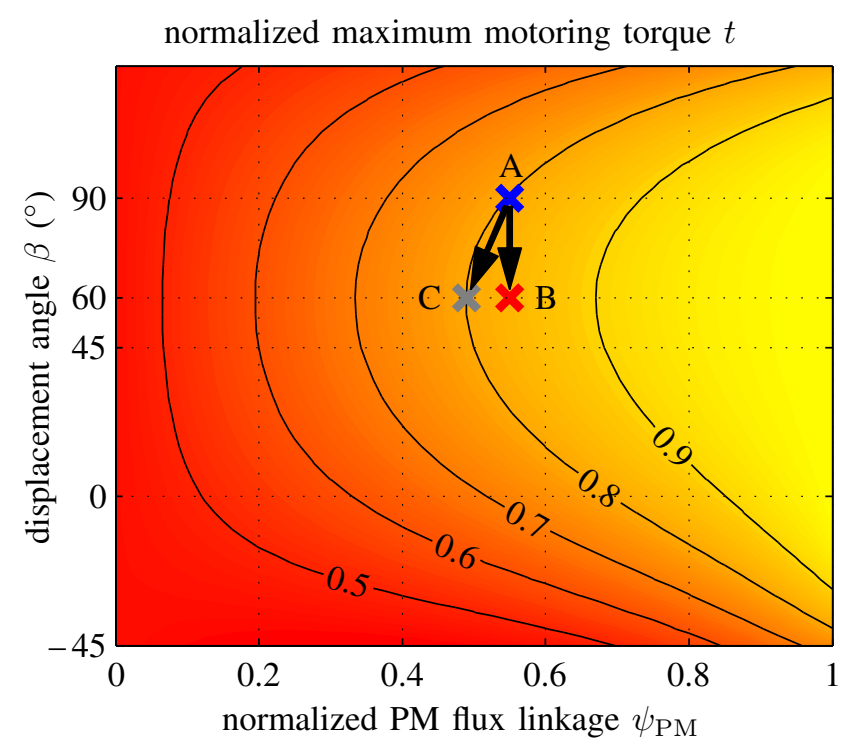

Fig. 2. Isolines of the normalized maximum motoring torque $t$ in the $\psi_{\mathrm{PM}^{-}}$ $\beta$-parameter plane for a fixed saliency value of $\zeta=4$ and exemplary machine designs (A-C).

mode occurs rather seldom and most energy is not recuperated in the base speed range at high torque values.

Nevertheless, the analysis in [4] has two limitations. First, the calculations are carried out for lossless ideal linear machines, so that important real-world effects such as losses and especially iron saturation are neglected. Second, there is no study on how to realize such a rotor except for a two part design as in [6].

This paper aims at these limitations by investigating finite element analysis (FEA) results of different machines, which are all based on a stator of a four pole industry motor with $5 \mathrm{~kW}$ nominal output power. Starting with real-world designs of a PMSM and an SPSM in Section II, it will be shown that pure PMSM and SPSM designs are inconvenient to be converted to machines with an arbitrarily displaced reluctance axis. In the second step, a hybrid synchronous machine concept with an appropriately displaced reluctance axis (HSM/DRA) will be deduced in Section III. Finally, Section IV discusses the performance and characteristic advantages of this machine concept in comparison to conventional machine concepts.

\section{Single Excitation Concepts}

\section{A. Permanent Magnet Synchronous Machines (PMSM)}

As discussed in the previous section, conventional PMSM with $L_{\mathrm{d}}<L_{\mathrm{q}}$ have a displacement angle of $\beta=90^{\circ}$. The physical cause of this angle lies in the machine geometry and the nature of permanent magnets, whose magnetic permeability is close to that of air. A pole pair of a simplified example machine is given in Fig. 3. The PM are usually buried in the rotor core for several reasons [1]. To avoid a magnetic short circuit, cavities must be positioned at the magnet sides which extend to the air gap to force the magnetic flux to enter

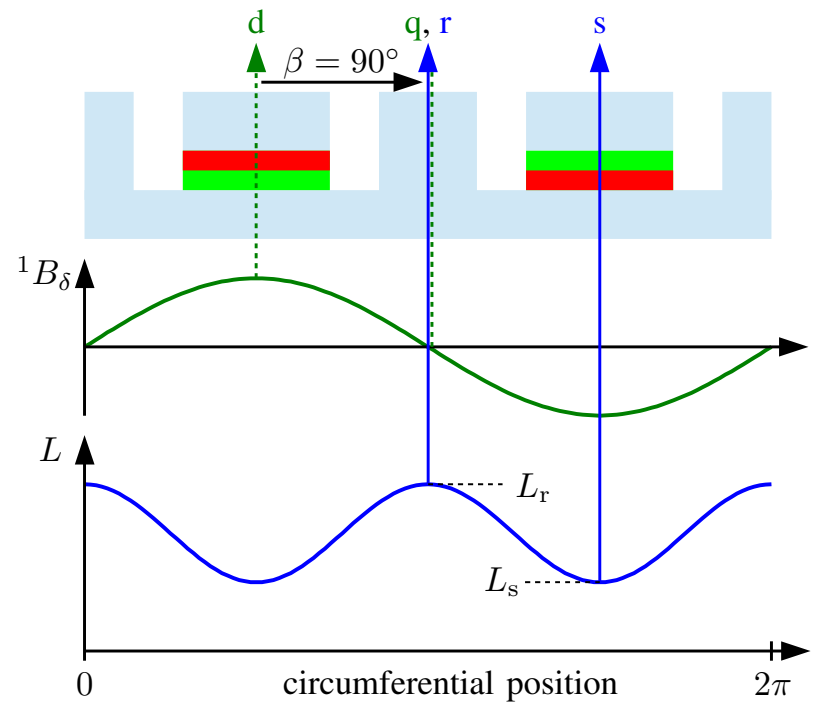

Fig. 3. Simplified geometry of a conventional PMSM, fundamental of air gap flux density ${ }^{1} B_{\delta}$ and inductance distribution $L$ versus the circumferential position and reference frames.

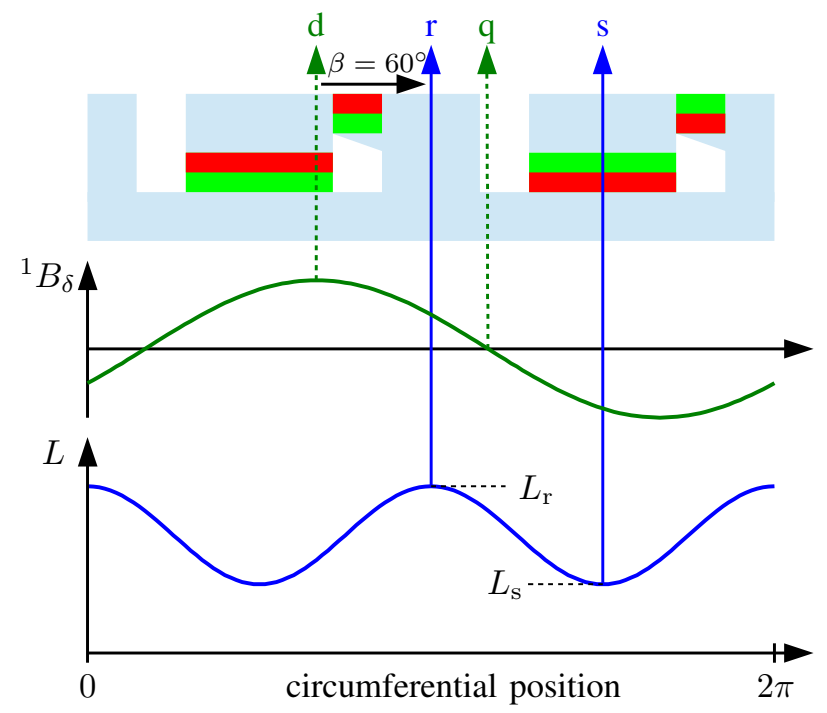

Fig. 4. Simplified rotor geometry of a PMSM with displaced reluctance axis, fundamental of air gap flux density ${ }^{1} B_{\delta}$ and inductance distribution $L$ versus the circumferential position and reference frames.

the stator. This structure automatically yields to a very low direct inductance, as the magnetic flux has to cross either the magnet or the cavities, which have both a very low magnetic permeability and a considerable magnetic length. However, the quadrature inductance remains high because there are unaffected flux paths in the rotor tangential to the magnets.

In order to improve the machine performance, the displacement angle $\beta$ should be lowered by about $30^{\circ}$ [4]. Unfortunately, simply moving the magnets sideways in their pockets or including additional magnets somewhere inside the rotor does not solve this problem because the actual position where the flux leaves the rotor on its surface does not change. Also, changing the reluctance structure does not help very 


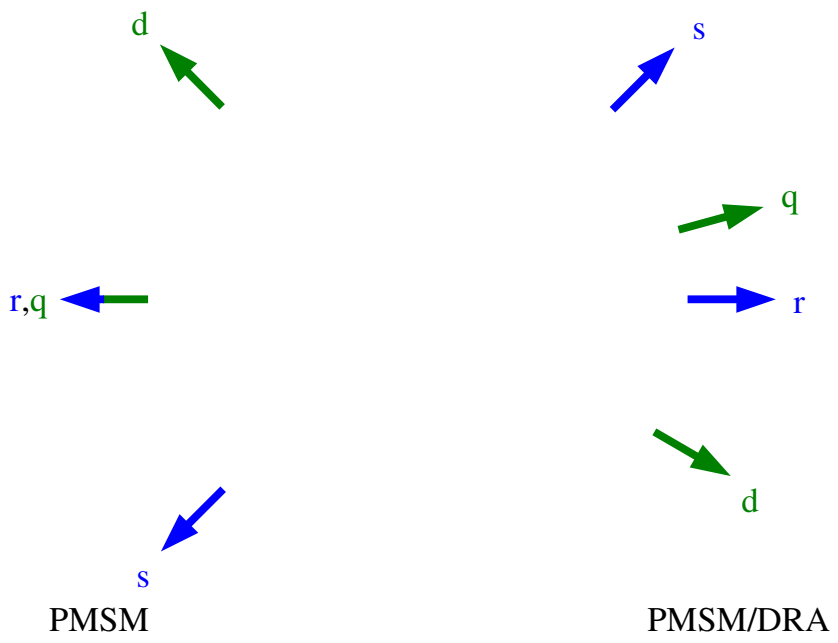

Fig. 5. Geometries of the conventional PMSM (left hand side) and the PMSM with displaced reluctance axis (right hand side). Cyan: stator and rotor iron; red and green: permanent magnets.

much as the PM flux always spreads homogeneously on the rotor surface, leaving the displacement angle at about $\beta=90^{\circ}$. Hence, the magnetic flux has to be manipulated directly on the rotor surface.

A possibility to achieve $\beta<90^{\circ}$ is given in Fig. 4. Magnets on the rotor surface are used to contribute a part of the air gap flux apart from the original d axis. They are located at the rotor cavities as this area has no radial flux components. Small iron triangles are used to carry the magnetic flux on the inner side of the magnets. Comparing Fig. 3 with Fig. 4, the fundamental of the air gap field is shifted closer to the $r$ axis whereas the direction of the inductance system is not affected.

Accordingly a PMSM with displaced reluctance axis (PMSM/DRA) has been designed and compared with a conventional PMSM (cPMSM). The geometries of these machines are depicted in Fig. 5. A rotor geometry with two layers was chosen to improve the saliency and to provide multiple positions for the surface magnets. The width of these magnets must be chosen carefully, the narrower they are, the greater is the relative stray flux, the wider they are, the more compromised are the flux paths in the $r$ axis, resulting in a lower $L_{\mathrm{r}}$ value.

Carrying out a FEA, two severe disadvantages of this concept could be identified. First, further harmonics are introduced in the open loop air gap field. Total harmonic distortion deteriorates from $4.3 \%$ to $29.5 \%$. This increases the harmonic content of the back electromotive force and the iron losses dramatically.

The second and even more serious effect is that due to the reduction of the displacement angle $\beta$, a certain amount of PM flux linkage flows through the reluctance part of the machine in the direction of the $r$ axis. This represents a "presaturation" of the reluctance axis, decreasing the ratio $\zeta=\frac{L_{\mathrm{r}}}{L_{\mathrm{s}}}$ of inductances due to the nonlinear nature of iron.
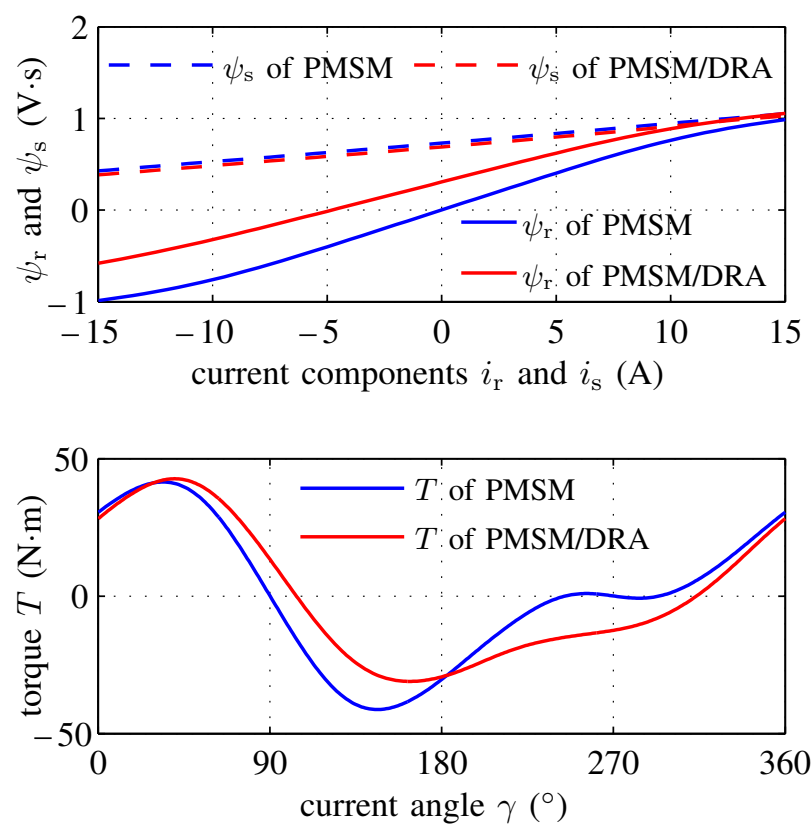

Fig. 6. Upper diagram: Flux linkages $\psi_{\mathrm{r}}\left(i_{\mathrm{r}}, i_{\mathrm{S}}=0 \mathrm{~A}\right)$ (solid lines) and $\psi_{\mathrm{s}}\left(i_{\mathrm{r}}=0 \mathrm{~A}, i_{\mathrm{s}}\right)$ (dashed lines) of the conventional PMSM (blue lines) and the PMSM with displaced reluctance axis (red lines).

Lower diagram: Torque of the conventional PMSM (blue lines) and the PMSM with displaced reluctance axis (red lines) over the current angle $\gamma$.

Fig. 6 shows the FEA results. In the upper diagram, flux linkages in $\mathrm{r}$ and $\mathrm{s}$ direction of both machines are plotted. Although cross saturation [7] has been considered in this paper, Fig. 6 only shows the flux linkage components with the other current fixed at $0 \mathrm{~A}$ for the sake of clarity, i.e. $\psi_{\mathrm{r}}\left(i_{\mathrm{r}}, i_{\mathrm{s}}=0 \mathrm{~A}\right)$ and $\psi_{\mathrm{s}}\left(i_{\mathrm{r}}=0 \mathrm{~A}, i_{\mathrm{S}}\right)$. The displacement of the excitation axis becomes apparent as an offset of the solid red $\psi_{\mathrm{r}}$-line at $i_{\mathrm{r}}=i_{\mathrm{s}}=0 \mathrm{~A}$. The $\mathrm{s}$ axis inductance $L_{\mathrm{s}}=\frac{\partial \psi_{\mathrm{s}}}{\partial i_{\mathrm{s}}}$ of both machines is similar as both traces are fairly parallel, yet the $r$ axis inductance of the machine with the displaced axis is smaller. As both traces converge to the same maximum value of flux linkage when increasing current, the effect of a decreasing saliency can be clearly blamed on saturation.

The lower diagram shows the torque of the cPMSM and the PMSM/DRA over the current angle $\gamma$. The PMSM/DRA achieves a slightly greater torque of $42.6 \mathrm{~N} \cdot \mathrm{m}$ as opposed to the cPMSM, which achieves $41.4 \mathrm{~N} \cdot \mathrm{m}$. As expected, generating performance of the PMSM/DRA is clearly worse $(-31.1 \mathrm{~N} \cdot \mathrm{m}$ vs. $-41.4 \mathrm{~N} \cdot \mathrm{m})$.

The designs can be evaluated easily after linearization and normalization, although the former introduces a slight error. Table I summarizes absolute and normalized values of both machines. Obviously, saturation decreases the inductance $L_{\mathrm{r}}$ and so the saliency $\zeta$ of the PMSM/DRA by about 20\%. PM flux linkage was increased by a few percent by applying more PM material in order to achieve the same normalized PM flux linkage. The gain in normalized motoring torque is only marginal. 
TABLE I

ABSOLUTE AND NORMALIZED MACHINE DATA. THE PMSM/DRA IS DESIGNED TO ACHIEVE EQUAL NORMALIZED PM FLUX LINKAGE

\begin{tabular}{lll}
\hline & cPMSM & PMSM/DRA \\
\hline r axis inductance $L_{\mathrm{r}}$ & $74.6 \mathrm{mH}$ & $59.7 \mathrm{mH}$ \\
s axis inductance $L_{\mathrm{s}}$ & $20.2 \mathrm{mH}$ & $20.8 \mathrm{mH}$ \\
absolute PM flux linkage $\psi_{\mathrm{PM}}$ & $0.729 \mathrm{~V} \cdot \mathrm{s}$ & $0.752 \mathrm{~V} \cdot \mathrm{s}$ \\
rated speed $n$ & $1404 \mathrm{~min}^{-1}$ & $1375 \mathrm{~min}^{-1}$ \\
\hline normalized PM flux linkage $\psi_{\mathrm{PM}}$ & 0.66 & 0.66 \\
saliency $\zeta=\frac{L_{\mathrm{r}}}{L_{\mathrm{s}}}$ & 3.70 & 2.86 \\
displacement angle $\beta$ & $90^{\circ}$ & $66^{\circ}$ \\
\hline normalized motoring torque $t$ & 0.870 & 0.877 \\
normalized generationg torque $t$ & -0.870 & -0.641 \\
\hline
\end{tabular}

normalized maximum motoring torque $t$

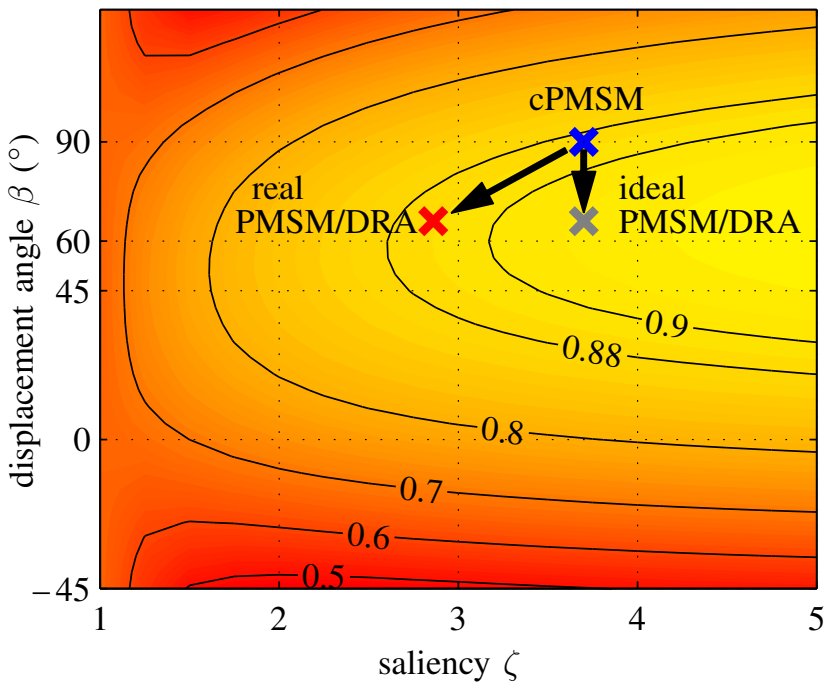

Fig. 7. Isolines of the normalized maximum motoring torque $t$ in the $\zeta-\beta$ parameter plane for a fixed saliency value of $\psi_{\mathrm{PM}}=0.66$ and the discussed machine designs.

The effect of saturation can be studied in more detail using the $\zeta$ - $\beta$-parameter plane (constant $\psi_{\mathrm{PM}}$ ) in Fig. 7 . Note that the values do not match exactly the values of Table I as an error due to linearization is introduced. The cPMSM design is marked with a blue cross. According to the linear theory [4], reducing the displacement angle to $\beta=66^{\circ}$ (the design moves to the gray cross), yields a normalized torque $t \approx 0.91$. However, due to saturation, saliency deteriorates and so does motor performance (red cross). This cancels out the positive effect of the displaced reluctance axis.

Obviously, it is not useful to apply displaced reluctance axis to PMSM designs.

\section{B. Salient Pole Synchronous Machines (SPSM)}

The theory of the displaced reluctance axis can also be applied to SPSM which have an adjustable excitation. SPSM with $\beta=0^{\circ}$ benefit from an increase of the displacement angle $\beta$, see Figs. 2 and 7. Starting from an arbitrary position on the $\beta=0^{\circ}$ line, torque increases when $\beta$ is raised and $\zeta$ is kept constant (i.e. moving upwards in the diagrams). As a sec-

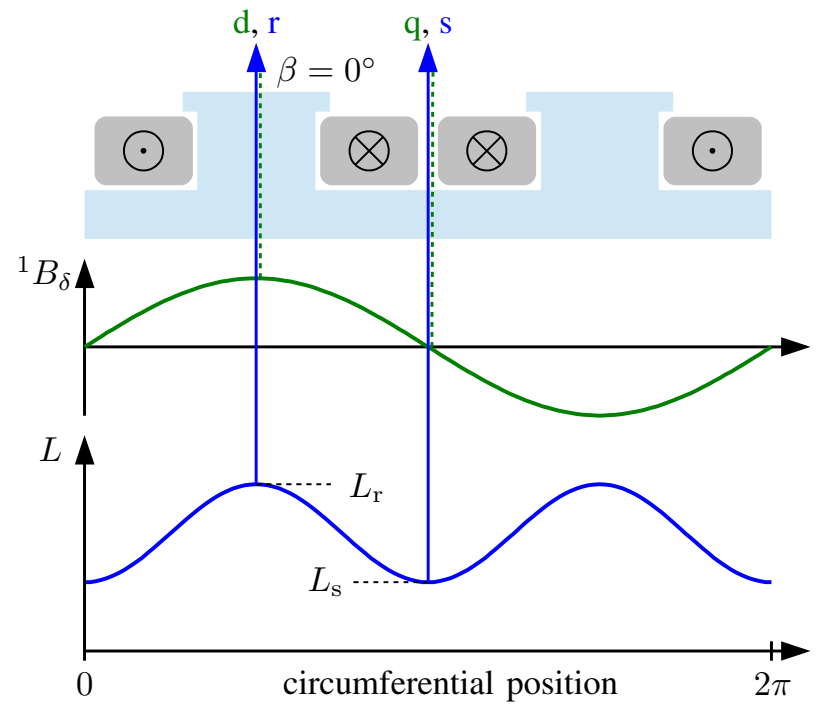

Fig. 8. Simplified rotor geometry of a conventional SPSM, fundamental of air gap flux density ${ }^{1} B_{\delta}$ and inductance distribution $L$ versus the circumferential position and reference frames.

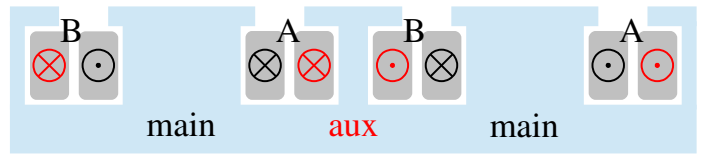

Fig. 9. Auxiliary poles trying to create a displaced reluctance axis.

ond benefit, regarding the saturation effect which deteriorates the PMSM performance by reducing saliency, increasing the displacement angle $\beta$ promises further improved performance as saturation in the reluctance axis will be reduced and thus saliency will be increased. Hence, applying a displaced reluctance axis to SPSM appears to be very promising.

Fig. 8 shows a simplified rotor geometry of an SPSM. Excitation is evoked by the field winding. Therefore, the permeability of the $\mathrm{d}$ axis must be as great as possible to achieve a high value of rotor flux linkage. The coil sides of the field windings lie in the $\mathrm{q}$ axis and, due to the physical properties of copper, lead to a comparatively low q axis inductance, although wide pole shoes can increase it.

The PMSM/DRA in the preceding subsection is created by additional magnets at the rotor surface. Equivalent to this approach, the geometry suggested in Fig. 9 incorporates small auxiliary poles to drag the fundamental of the air gap flux density away from the $\mathrm{r}$ axis. Using this method, several problems emerge: First, these poles increase the inductance in the $\mathrm{q}$ axis as the copper in this axis is partially replaced by iron, decreasing saliency. Second, there are opposing coil sides in every other slot (indicated with "B"), whose impact cancels each other out. Assuming identical turn number, coil sides in these slots can therefore be omitted as well. This however leads to a dramatically decreased usable slot area, leading to high rotor copper losses. Third, the $\mathrm{d}$ axis would move towards the auxiliary pole as intended, but depending on 


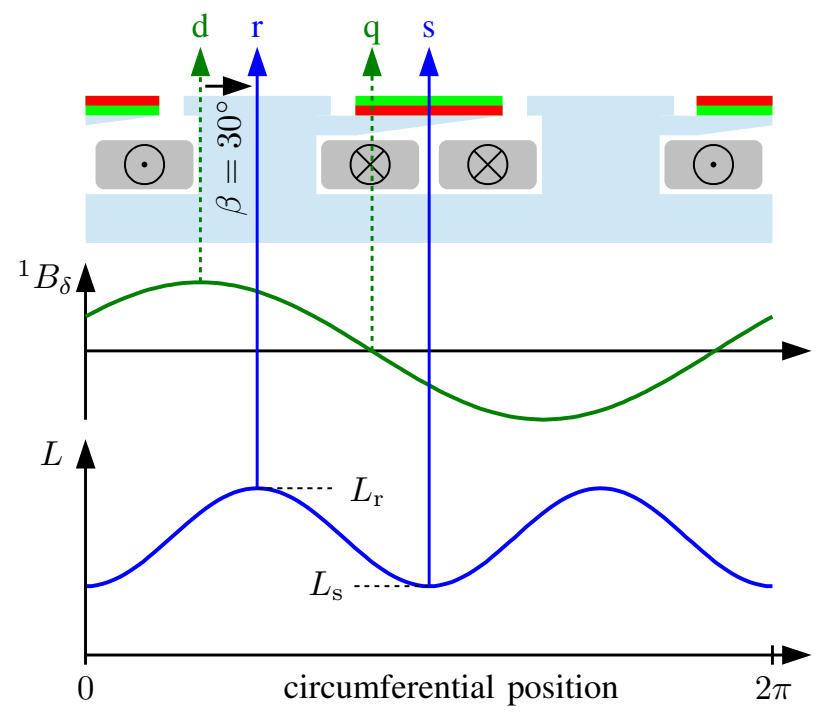

Fig. 10. Basic rotor geometry of the proposed hybrid excitation machine (HSM/DRA), fundamental of air gap flux density ${ }^{1} B_{\delta}$ and inductance distribution $L$ versus the circumferential position and reference frames.

the geometry, the reluctance axis would move as well, leading to no significant change in displacement angle.

In summary, because rotor excitation and armature reaction are both generated by electric current and therefore rely on the same physical conditions, it is not possible to implement an independently displaced reluctance axis to SPSM designs.

\section{A HyBRid EXCITATION CONCEPT WITH DisPlaCED RELUCTANCE AXIS (HSM/DRA)}

As it becomes clear in the preceding section, the auxiliary poles of the attempt to introduce a displaced reluctance axis to an SPSM must be of low magnetic permeability, so that the $\mathrm{d}$ axis gets shifted whereas the reluctance structure is not affected. Therefore, a hybrid excitation concept is proposed, which can be considered as an SPSM with auxiliary PM poles, see Fig. 10. The excitation winding creates a certain amount of rotor flux which is located in the d axis, whereas surface PM contribute in the low inductance axis, just like the surface magnets of the PMSM/DRA.

The magnetic flux of the PM on the inner side of the rotor is carried by triangular arms and is led to the adjacent salient poles, which show the opposite magnetic polarity on their surface. This has the effect that only a certain fraction of the rotor flux is carried by the salient poles and the rotor yoke. This leads to lower saturation and the saliency increases, which promises greater reluctance torque.

Interestingly, a very similar geometry has been proposed in [8] as a generator for automobiles, where the PM are used to increase the air gap field. Although the inventors have realized the saturation advantage of this geometry, the benefits of the displaced reluctance axis have obviously not been utilized.

As opposed to conventional hybrid synchonous machines, which are collected and compared in [9] and [10], this geometry maintains its relatively high saliency as there are
SPSM

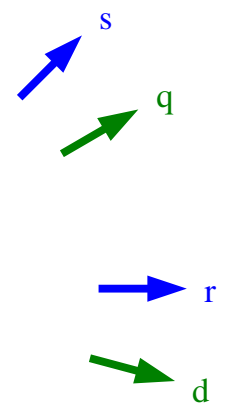

PMSM

HSM/DRA

Fig. 11. Proposed hybrid excitation geometry (right hand side), which is compared to an SPSM (upper left hand side) and to the previously introduced PMSM (lower left hand side). Cyan: stator and rotor iron; red and green: permanent magnets; gray: excitation winding.

no magnets in the reluctance axis. Series hybrid synchronous machines incorporate $\mathrm{PM}$ in the $\mathrm{d}$ axis of the excitation system, hence corrupting both saliency and the effectiveness of the excitation due to the increased air gap. Parallel hybrid synchronous machines usually have different poles with alternating PM and electric excitation, which also leads to a low overall saliency.

\section{Performance Analysis}

In order to analyze the proposed geometry and to compare it with conventional PMSM and SPSM designs, three machines have been designed. Their geometries are shown in Fig. 11 . They all use the same four pole stator geometry, whose data is given in Table II. The PMSM geometry is the same that was already used in Section II. The hybrid synchronous machine with displaced reluctance axis (HSM/DRA) and the SPSM are designed with equal gross rotor slot area and calculated with the same value for rotor resistance. As the PM part of the HSM/DRA requires additional space, the rotor yoke and salient poles are slightly narrower compared to those of the SPSM. This does not lead to disadvantages because the total flux in this parts is lower as described earlier.

The geometry of the HSM/DRA has been optimized with respect to electromagnetic behavior. However, manufacturing

TABLE II

BASIC DATA OF THE COMMONLY USED STATOR

\begin{tabular}{ll}
\hline active length $l_{\mathrm{Fe}}$ & $160 \mathrm{~mm}$ \\
stator inner diameter $D_{\mathrm{i}}$ & $110 \mathrm{~mm}$ \\
rated RMS line-line voltage $V_{\mathrm{N}}$ & $400 \mathrm{~V}$ \\
rated RMS line current $I_{\mathrm{N}}$ & $10 \mathrm{~A}$ \\
rated speed $n_{\mathrm{N}}$ & $1500 \mathrm{~min}^{-1}$ \\
\hline
\end{tabular}




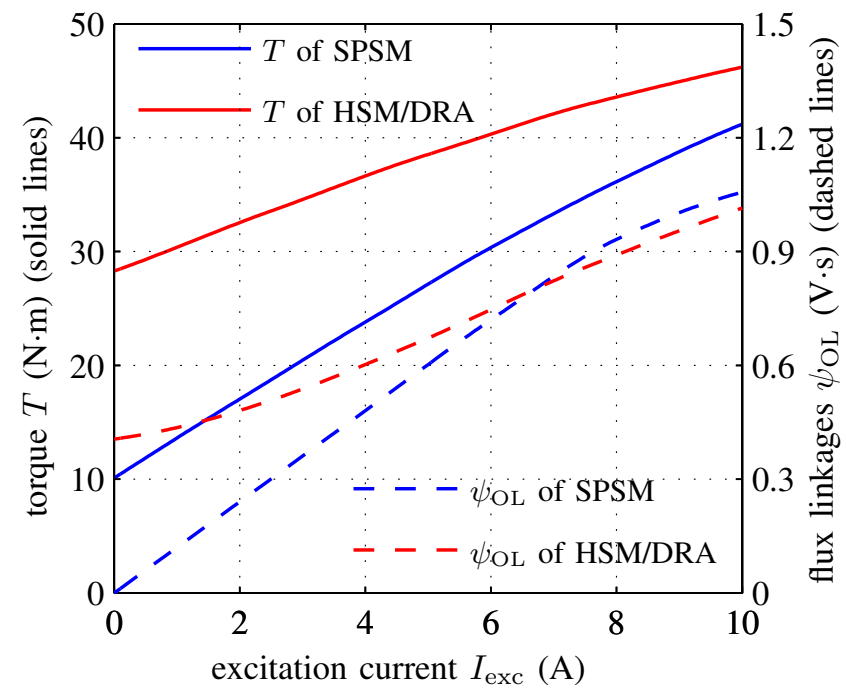

Fig. 12. Solid lines: Maximum torque $T$ depending on the excitation current $I_{\text {exc }}$ at stator current $I=10 \mathrm{~A}$ and optimum current angle. Dashed lines: No load rotor flux linkage $\psi_{\mathrm{OL}}$. Blue lines: SPSM. Red lines: HSM/DRA.

of this geometry would be a challenge as the rotor slots are entirely covered with PM and flux carrying iron parts. In order to simplify assembly, the geometry should be revised.

\section{A. Effect of the Displaced Reluctance Axis}

To investigate the positive influence of the displaced reluctance axis, the maximum achievable torque at rated stator current $(I=10 \mathrm{~A})$ and at optimum current angle depending on the excitation current $I_{\text {exc }}$ and the open loop flux linkage $\psi_{\mathrm{OL}}=f\left(I_{\text {exc }}\right)$ based on FEA results for both the SPSM and the HSM/DRA are plotted in Fig. 12. As the structure of the HSM/DRA and the SPSM are similar and the PMSM has no adjustable excitation, only the former two machine concepts are compared in this subsection.

At first, it must be noted that the PM of the HSM/DRA create an offset in $\psi_{\mathrm{OL}}$ at $I_{\mathrm{exc}}=0 \mathrm{~A}$, whereas the SPSM starts at $0 \mathrm{~V} \cdot \mathrm{s}$. Increasing current leads to a linear increase in rotor flux linkage of the SPSM until approximately $7 \mathrm{~A}$, where saturation becomes noticeable. Rotor flux linkage of the HSM/DRA starts to increase at a slow rate initially as the excitation winding becomes effective in the $r$ axis, whereas the $\mathrm{PM}$ are located in the s axis. The slope at greater excitation currents is still not as steep as the slope of the SPSM because the salient pole surface at the air gap of the HSM/DRA is narrower.

At $I_{\text {exc }}=0 \mathrm{~A}$, both machines are able to supply torque because of the saliency. The HSM/DRA is also able to utilize the PM flux linkage, which is why its torque is considerably greater. Starting at $I_{\text {exc }} \approx 7 \mathrm{~A}$, the SPSM creates greater rotor flux linkage, yet the HSM/DRA generates significantly more torque, which proves the conceptual benefits of this machine.

As a side note, the HSM/DRA starts at $\beta=90^{\circ}$ when $I_{\text {exc }}=0 \mathrm{~A}$. This is because the PM are located in the s axis and the electrical excitation is not yet active. As the excitation current increases, the displacement angle decreases until it reaches $\beta \approx 30^{\circ}$ when $I_{\mathrm{exc}}=10 \mathrm{~A}$.

\section{B. Efficiency}

Not only does the HSM/DRA produce the greatest maximum torque, it also requires less excitation current to achieve a desired torque, which promises efficiency benefits. In order to investigate this aspect in more detail, efficiency maps of the three machines have been calculated, using a procedure similar to [11]. Flux linkages and normalized iron losses of the three machines depending on the three variables stator current magnitude $I$, stator current angle $\gamma$ and excitation current $I_{\text {exc }}$ have been calculated using FEA. Best efficiency in all possible operating points has been found by numerical optimization. In this process, copper, iron, magnet and friction losses have been considered. The results are given in Figs. 13 to 15 .

Fig. 13 shows the efficiency map of the PMSM. It supplies good efficiency at low speed and low torque and its peak efficiency of $\eta_{\max }>95 \%$ is the greatest of all three machines, yet efficiency decreases at higher speeds as a demagnetization current is required for field weakening. Due to the rather low electric loading of the stator of $A \approx 24 \frac{\mathrm{A}}{\mathrm{mm}}$, field weakening capability is low so that there is a maximum speed of $n_{\text {max }} \approx 3700 \mathrm{~min}^{-1}$ which can not be exceeded. However, this limitation is due to the electromagnetic design of the machine. Using less PM material, the maximum speed can be increased, yet decreasing maximum torque in the base speed range.

Fig. 14 shows the efficiency map of the SPSM. It features excellent field weakening performance as its rotor excitation is adjustable. Its peak efficiency of $\eta_{\max }=92.6 \%$ is the lowest of all three machines, but efficiency remains quite high in the field weakening region. However, efficiency at low speeds is comparably poor because the excitation losses are dominant in this area.

Fig. 15 shows the efficiency map of the HSM/DRA. Due to the displaced reluctance axis, maximum motoring torque is significantly greater than the maximum generating torque and is the greatest of all three machines. Due to the partially adjustable excitation, performance in the field weakening region remains excellent. The peak efficiency of $\eta_{\max }=94.6 \%$ is close to that of the PMSM. Regarding overall efficiency, the machine combines the advantages of both, the PMSM and the SPSM: at low speeds and low torque, efficiency is high because the excitation current can be kept at a minimum. At high speeds, field weakening can be achieved by reducing the excitation current. Analysis of the optimized current values (not given in this paper) reveals that full excitation current is only necessary for highest torque in the base speed range as opposed to the SPSM, where full excitation current is also applied in the field weakening range.

Excitation current has been varied in the range of $0 \mathrm{~A} \leq$ $I_{\text {exc }} \leq 10 \mathrm{~A}$. An analysis covering the range $-10 \mathrm{~A} \leq I_{\text {exc }} \leq$ $10 \mathrm{~A}$ has been carried out but it did not reveal significant benefits as the magnetic circuit of the HSM/DRA is not designed for negative excitation current. 


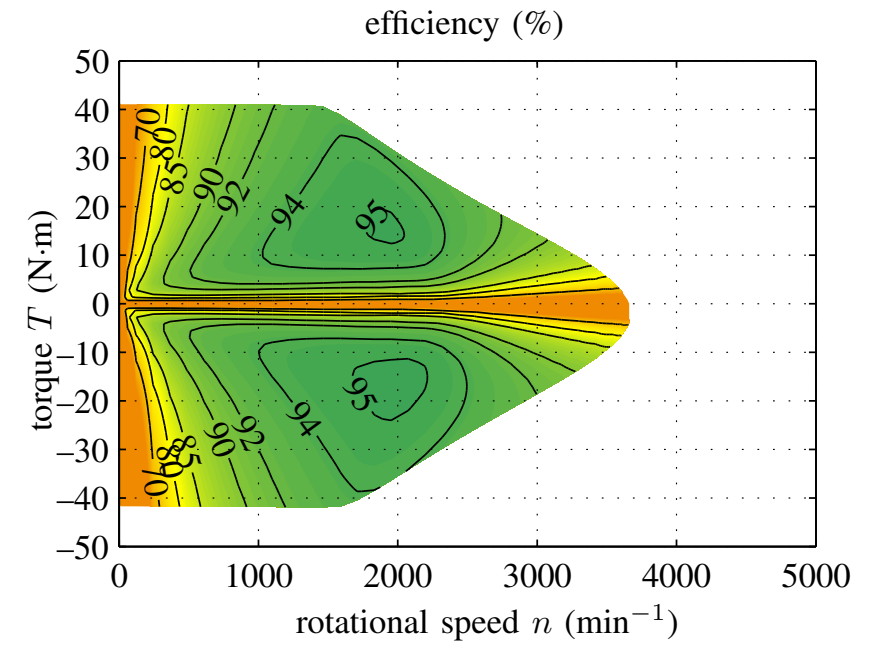

Fig. 13. Efficiency map of the PMSM. High peak efficiency, fair field weakening efficiency and maximum torque, low field weakening performance.

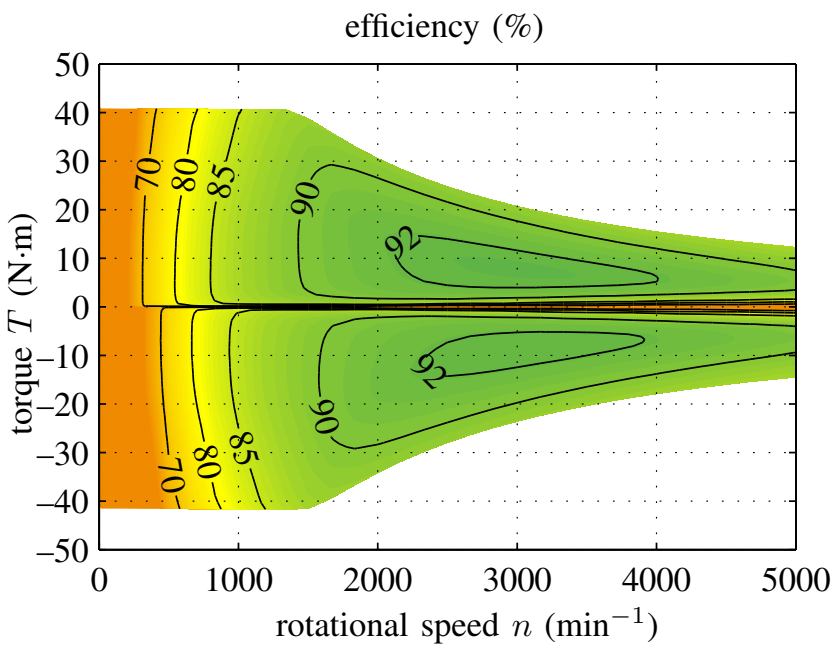

Fig. 14. Efficiency map of the SPSM. Low peak efficiency, good efficiency and performance in the field weakening range, fair maximum torque.

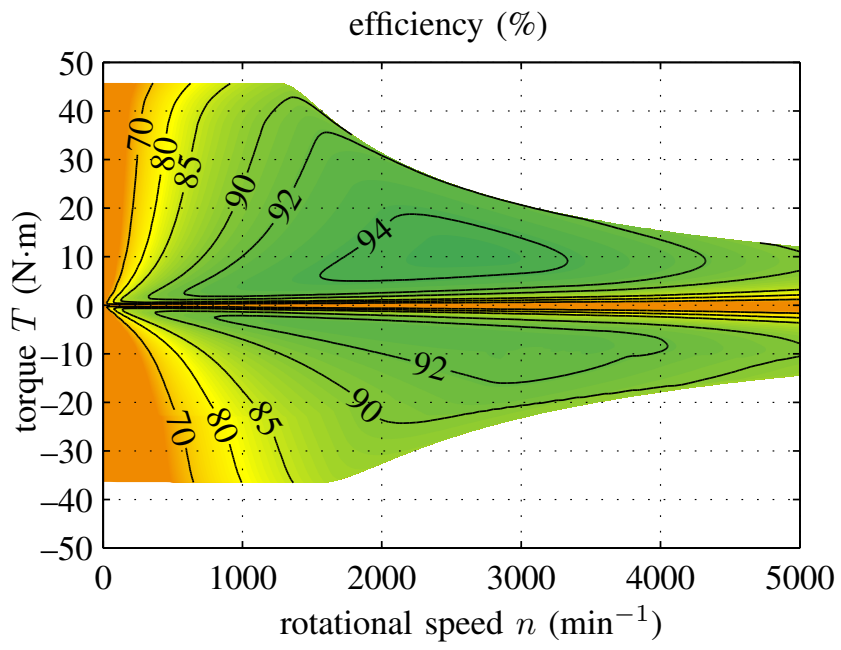

Fig. 15. Efficiency map of the HSM/DRA. Maximum torque, field weakening performance and efficiency are very good in motoring mode. Generating mode is inferior as a matter of principle.

\section{CONClusion}

In this paper, several possibilities to realize a synchronous machine with displaced reluctance axis have been discussed. It was shown that machines with single excitation - no matter if PM excited or current excited - are impossible to design usefully for a number of reasons. The geometry of a hybrid excitation machine with displaced reluctance axis has been deduced to avoid the identified disadvantages.

In the second part, several exemplary machines have been designed and analyzed. The superiority of the hybrid synchronous machine concept with displaced reluctance axis is shown: It combines the specific efficiency advantages of both PMSM and SPSM designs and shows superior performance in motoring mode.

\section{REFERENCES}

[1] T. Jahns, G. Kliman, and T. W. Neumann, "Interior permanent-magnet synchronous motors for adjustable-speed drives," Industry Applications, IEEE Transactions on, vol. IA-22, pp. 738-747, 1986.

[2] W. Soong and T. Miller, "Theoretical limitations to the field-weakening performance of the five classes of brushless synchronous AC motor drive," in Electrical Machines and Drives, 1993. Sixth International Conference on (Conf. Publ. No. 376), 1993, pp. 127-132.

[3] P. Winzer and M. Doppelbauer, "Charakterisierung und Auslegung von fremderregten Synchronmaschinen als Traktionsantrieb mit Hilfe der normierten Parameterebene," in Internationaler ETG-Kongress 2013 (ETG-FB 139), 2013.

[4] P. Winzer and M. Doppelbauer, "Theoretical analysis of synchronous machines with displaced reluctance axis," in Electrical Machines (ICEM), 2014 XXIth International Conference on, 2014, pp. 641-647.

[5] J. Pyrhonen, T. Jokinen, and V. Hrabovcova, Design of Rotating Electrical Machines. John Wiley \& Sons, Ltd, 2008.

[6] B. Chalmers, L. Musaba, and D. Gosden, "Variable-frequency synchronous motor drives for electric vehicles," Industry Applications, IEEE Transactions on, vol. 32, pp. 896-903, 1996.

[7] B. Stumberger, G. Stumberger, D. Dolinar, A. Hamler, and M. Trlep, "Evaluation of saturation and cross-magnetization effects in interior permanent-magnet synchronous motor," Industry Applications, IEEE Transactions on, vol. 39, pp. 1264-1271, 2003.

[8] F. Liang, M. Miller, and X. Xu, "Hybrid permanent magnet/synchronous machine," U.S. Patent 6,548,931 B2, 2003.

[9] Y. Amara, L. Vido, M. Gabsi, E. Hoang, A. Hamid Ben Ahmed, and M. Lecrivain, "Hybrid excitation synchronous machines: Energyefficient solution for vehicles propulsion," Vehicular Technology, IEEE Transactions on, vol. 58, pp. 2137-2149, 2009.

[10] S. Wang, S. Ni, Y. Xia, X. Wang, P. Su, and S. Huang, "Hybrid excitation permanent magnet synchronous machines and their structures," in Electrical Machines (ICEM), 2014 XXIth International Conference on, 2014, pp. 2606-2612.

[11] A. Daanoune, A. Foggia, L. Garbuio, J. Mipo, and L. Li, "Modeling and optimal control of a hybrid excitation synchronous machine by combining analytical and finite element models," in Electrical Machines (ICEM), 2012 XXth International Conference on, 2012, pp. 2448-2453.

Patrick Winzer was born in Lörrach, Germany in 1985. He graduated from Karlsruhe Institute of Technology (KIT) in 2011 and works since then at the Institute of Electrical Engineering at KIT as Ph.D. student in the field of electric traction drives.

Martin Doppelbauer studied electrical engineering at the University of Dortmund in Germany and graduated 1995 with a doctorate thesis on the analytical calculation of universal motors. He worked in the industry from 1995 until 2010. Since 2011 Martin Doppelbauer is full professor at the Institute of Electrical Engineering (ETI) of the Karlsruhe Institute of Technology (KIT) where he holds a chair for Hybrid Electric Vehicles. 